\title{
Early rehabilitation protocol after Achilles tendon tenorraphy: ultrasonography and elastosonography difference with immobilization in cast: an observational study.
}

\section{Mario Mosconi}

Universita degli Studi di Pavia Facolta di Medicina e Chirurgia

\section{Eugenio Jannelli}

Universita degli Studi di Pavia Facolta di Medicina e Chirurgia

Alberto Castelli

Universita degli Studi di Pavia Facolta di Medicina e Chirurgia

Enrico Ferranti Calderoni

Universita degli Studi di Pavia Facolta di Medicina e Chirurgia Jessica Zanovello ( $\sim$ jessica.zanovello@gmail.com )

Universita degli Studi di Pavia Facolta di Medicina e Chirurgia https://orcid.org/0000-0001-9317-2423

\section{Matteo Ghiara}

Universita degli Studi di Pavia Facolta di Medicina e Chirurgia

\section{Simone Perelli}

Universitat Autonoma de Barcelona

\section{Francesco Benazzo}

Universita degli Studi di Pavia Facolta di Medicina e Chirurgia

\section{Study protocol}

Keywords: Achilles tendon, Tenorraphy, Early rehabilitation, Ultrasonography, Elastosonography,

Posted Date: April 1st, 2020

DOI: https://doi.org/10.21203/rs.3.rs-19067/v1

License: (c) (i) This work is licensed under a Creative Commons Attribution 4.0 International License. Read Full License 


\section{Abstract}

Background The incidence of Achilles tendon (AT) rupture increased, but there is no gold standard treatment. The aim of this study is to compare clinical and functional results of standard and fast rehabilitation program (immobilization and no weight bearing for 4 weeks vs early joint mobility and weight bearing).

Methods 33 patients underwent to open achilles tenorraphy between January and July 2018 using Krackow suture. 15 patients were enrolled and underwent fast rehabilitation program. A control group of 18 patients underwent surgery and standard protocol. The degree of sural triceps hypotrophy compared with the controlateral leg (calf circumference $4 \mathrm{~cm}$ below the anterior tibial tuberosity and the tibial length), American Orthopaedic Foot and Ankle Society (AOFAS) Ankle-Hindfoot Score [8], the Victorian Institute of Sports Assesement - Achilles Questionnaire (VISA-A) [9] and The Achilles tendon Total Rupture Score (ATRS) [10] were recorded at 12 months follow up. Ultrasonography and elastosonography were used to calculate different parameters both on the operated tendon and on the contralateral.

Discussion Using ultrasonography, the only significant difference was recorded in the distal depth in both groups. We detected an increase of all measures in both groups comparing surgical side to the contralateral one. Using the elastosonography, the stiffness on healthy side was increased at the proximal and medial third in standard group, even if the distal third was major in fast group. On the operated side, stiffness was more on the proximal third in fast group (not statistically significant). There was an increase of tendon size on the surgical side compared to the "basal" one using US. The only statistically significant result was at distal third, and it could be due to the fact that giving an accelerated mobilization stimulates more collagen deposition, which could be a protective factor. Furthermore, at distal third of tendon we observed a greater reduction of stiffness in the fast group compared of the standard one. The results of the post-operative assessment scales are overlapping in the two groups. Clinical and functional evidence suggest that fast rehabilitation after AT surgical repair may be a first choice of treat, especially in young and active patients, because of minor local complications and higher level of satisfaction.

\section{Background}

Achilles tendon (AT) rupture is one of the most frequent injuries of tendons in middle age population [1].

The overall incidence per 100,000 person-years increased from 2.1 (95\% Cl 0.3-7.7) in 1979 to 21.5 (95\% $\mathrm{Cl} 14.6-30.6)$ in 2011 [1]. The incidence increased in all age groups. The mean annual increase in incidence was $2.4 \%$ (95\% $\mathrm{Cl} 1.3-4.7)$ higher for non-sports-related ruptures than for sports-related ruptures $(P=0.036)[1]$.

AT rupture used to occur in men in their $40 \mathrm{~s}$ (man to female ratio is approximately 6:1(109), but in a recent study it has been demonstrated that age and sex have not so important influence on AT ruptures as it was in the past due to an increased participations of elderly populations and females to sports [2] 
.The rupture of the Achilles' tendon is evidenced mostly in patients who occasionally perform physical activity, due to a precarious preparation for the physical effort. Ruptures are usually monolateral, $57 \%$ on the left side, $43 \%$ on the right side [3]; simultaneous ruptures are rare generally due to high altitude fall [4]

There is no gold standard treatment in literature for Achilles tendon rupture: conservative and surgical are both accepted $[5,6]$. There are advantages and disadvantages inherent each treatment. Conservative treatment is considered the best solution in elderly patients with co-morbid medical conditions and in patients with not high-performance demand. Surgical treatment is performed in most patients (95.6\%) [4].

There are different surgical methods. The decision concerning which is the best treatment is based on many different factors: acute, subacute or chronic lesions, age and way of living of the patient, comorbidities, experience of the surgeon and the economic implications (mini-invasive surgery is more expensive than open surgery, but both surgeries are more expensive than conservative treatment) $[4,7]$.

Furthermore the rehabilitation protocol after surgery is controversial in literature. However goals of treatment are prevention of secondary complication and recurrent lesion, early restoration of articular range of motion (ROM) and rapid recovery of strength and proprioception.

The aim of this prospective observational clinical study is to compare the clinical and functional results of a standard rehabilitation protocol which consists in the immobilization in splint and no weight bearing for 4 weeks, and a fast rehabilitation program, which provides an early restoration of joint mobility with a fast recovery of full weight bearing.

\section{Methods}

33 patients underwent to open achilles tenorraphy at our institution by single senior surgeon between January 2018 and July 2018.

All patients were placed in a prone position with feet protruding from the table with tourniquet placed on the thigh. We used a posterolateral approach in all cases to have a direct visualization of the sural nerve, thus avoiding involuntary lesions of the same. The two ends of the lesion were armed with Krackow suture with 4 loops blocked on each side of the lesion and 4 side-to-side stitches with vicryl $2-0$. A crossstitch with 0-PDS (Ethicon, Somerville, NJ, USA) was used to reinforce the suture. If the plantar tendon is present and not damaged, it can be used as an autograft for the same purpose. The limb was bandaged in equine bland with vascular elastic bandages.

We excluded patients older than 65 years old or recurrent lesion with previous physical or surgical therapy, postoperative complications, rheumatic disease or collagenopathies, third proximal or distal lesion, delayed surgery more than ten days after injury, patients who refusal to accept the proposed fast rehabilitation program (Table 1).

Of 33 patients, only 15 patients presented the required features, and they were enrolled. 
The control group is composed of 18 patients with similar features in terms of age, site of injury and functional requests, which underwent surgery and standard rehabilitation protocol (table 2).

\section{Table.1 Fast Rehabilitation Protocol}

0-7 Bland equinus bandage with elastic vascular band, passive and active mobilization from

days $90^{\circ}$ of dorsiflextion to maximum plantar flexion, walking with crutches out of charge on the operated limb

7-45 Replace bandage with orthotic device during the weight bearing, a plastic boot-shaped brace days which aim is to block the ankle movement in dorsiflexion, allowing the healing of surgical suture. $1 \mathrm{~cm}$ wedges are placed inside the brace, depeding on muscular and tendon tension reached after surgery. Every 10 days, one wedge is removed. During this period, a progressive increase of the weight bearing is required. The brace has to be removed several times a day to mobilize the ankle.

$>45$ Progressive abandonment of the brace and starting isometric strengthening of sural triceps days

$>60 \quad$ Starting concentric strengthening of sural triceps

days

$>12$ Starting stretching of sural triceps and gradual resumption of the race if the recovery of weeks ROM of the ankle and muscle tropism is complete.

\section{Table.2 Standard Rehabilitation Protocol}

0-5 Plaster splint in equinus position until the first clinic control.

days

5-21 Immobilization in equinus cast, walking with crutches out of charge on the operated limb days

21- Replace equinus plaster splint with orthomorphic cast for 3-8 weeks (depending on surgical 45 complications)

days

$>45$ Walking with crutches with partial weight-bearing allowed, starting FKT of passive and active days mobilization of the ankle, avoiding maximal dorsiflexion for two weeks

$>60$ Isometric and concentric strengthening of sural triceps

days

$>75$ Walking with complete load, starting Stanish exercises and eccentric strengthening. days

All patients were preoperatively evaluated with clinical and ultrasonographic exam, to have a preoperative data concerning the site and the type of lesion.

Moreover, patients' anamnestic data were collected: age, BMI, works, previous injury, surgery, sports participation, and previous therapies on the tendons. Furthermore, we intraoperatively recorded plantar tendon ruptures and insertional calcaneus deformities such as Haglund's disease. 
All patients were clinically evaluated at 12 months follow up. Clinical parameters were used to test the degree of sural triceps hypotrophy compared with the controlateral leg: calf circumference $4 \mathrm{~cm}$ below the anterior tibial tuberosity and the tibial length measured from the anterior tibial tuberosity to the medial malleolus. Moreover, we used American Orthopaedic Foot and Ankle Society (AOFAS) Ankle-Hindfoot Score [8], the Victorian Institute of Sports Assesement - Achilles Questionnaire (VISA-A) [9] and The Achilles tendon Total Rupture Score (ATRS) [10] at the follow-up.

Furthermore, ultrasonography (Esoate MyLAb 70 ultrasound scanner with a $7.5 \mathrm{MHz}$ linear transduce) and elastosonography (Esaote MyLAb 70 with a $7.5 \mathrm{MHz}$ linear transducer, using the Strai methodology), performed by a single sonographer, were used to calculate different parameters both on the operated tendon and on the contralateral.

During US both the depth/thickness of the tendon (ventro-dorsal diameter) and the width (medium-lateral diameter) were calculated. These two measurements were collected along the tendon at three levels: proximal (musculotendinous junction), medium (2-6 cm above the insertion in the calcaneus) and distal (insertion at the heel), thus obtaining six values for each tendon.

Concerning ES, using the B-mode ultrasound display as a guide, three regions of interest (ROI) were selected at the same point in which ultrasound measurements were taken. In these regions, the percentage of red colour in the colour scale, which represents the elasticity values, was evaluated so as to obtain the percentage of tendon stiffness at myotendinous level, at the middle third (the region of the rupture in the operated tendon) and at distal level in the calcaneal insertion region.

Healthy limb measurements were considered as a "baseline" or reference value: for all measurements the difference between the one made on the injured limb after the operation and on the healthy limb was calculated, thus obtaining values on which the statistical analysis was performed.

Descriptive statistics were used for all demographic and subjective data, sports participation, and rehabilitation data. Continuous variables were presented with average and standard deviation (SD). Categorical variables were presented as frequency and percentages, or median and $25^{\circ}$ e $75^{\circ}$ percentile. To analyse the differences between standard and accelerated rehabilitation program data, Mann-Whitney test was applied to normally distributed continuous outcomes, while chi-square test or Fisher test were used for categorical outcomes. For all tests, a p-value less than 0.05 was considered statistically significant. All statistical analyses were performed using Microsoft Excel.

\section{Results}

15 patients who underwent fast rehabilitation protocol were men with an average age of 43.1 years (SD 7.1). 8 suffered ruptures on the right side and 7 on the left side. The average of the waiting time between injury and surgery was 5.9 days (range 1-10 days). No intraoperative or postoperative complications were observed, such as infection or deep venous thrombosis. 
18 patients were treated by standard rehabilitation program after surgery. All of these patients were men with an average age of 38.4 years (SD 9.9) and valuated at 1 year follow-up. 12 suffered ruptures on the right side and 6 on the left side.

As it is showed in the Table 3, we analyzed some features in both groups: the differences between two groups were not statistically significant.

Table 3

Patients features

\begin{tabular}{|llll|}
\hline & $\begin{array}{l}\text { Standard rehab. } \\
\mathbf{N}=\mathbf{1 8}\end{array}$ & $\begin{array}{l}\text { Fast rehab. } \\
\mathbf{N}=\mathbf{1 5}\end{array}$ & P-Value \\
\hline Age (years) & $38.4(9.9)$ & $43.1(7.1)$ & 0.11 \\
\hline Sedentary job (N, \%) & $11(46.7 \%)$ & $7(61.1 \%)$ & 0.41 \\
\hline Sports (N, \%) & $12(66.7 \%)$ & $13(86.7 \%)$ & 0.18 \\
\hline Haglund (N,\%) & $18(100 \%)$ & $14(93.3 \%)$ & 0.46 \\
\hline Plantaris tendon rupture (N, \%) & $0(0 \%)$ & $1(6.7 \%)$ & 0.46 \\
\hline Flat foot (N, \%) & $2(11.1 \%)$ & $1(6.7 \%)$ & 0.57 \\
\hline No pharmacological therapy (N, \%) & $16(88.9 \%)$ & $9(60 \%)$ & 0.06 \\
\hline Calf circumference (cm, SD) & $36.9(2,5)$ & $36.3(\mathrm{SD} 2.6)$ & 0.50 \\
\hline Tibial length (cm, SD) & $35.0(1.8)$ & $36.0(1.9)$ & 0.19 \\
\hline
\end{tabular}

We used ultrasound and elastosonography to analyse differences between healthy and operated tendon in the two groups.

There are no US differences in depth and length in healthy tendon (considered as "basal state") in the proximal and medial part, although we detected a significant difference in the distal depth in both groups (Table 4). 
Table 4

Ultrasound measurements on healthy tendon

\begin{tabular}{|llll|}
\hline & $\begin{array}{l}\text { Standard rehab. } \\
\mathbf{N}=18\end{array}$ & $\begin{array}{l}\text { Fast rehab. } \\
\mathbf{N}=15\end{array}$ & Average and P-value \\
\hline Proximal width (mm, SD) & $21(4.6)$ & $22.5(5)$ & $\mathrm{MW}=-0.36, \mathrm{p}=0.72$ \\
\hline Proximal depth (mm, SD) & $2.4(0.7)$ & $3.0(1.3)$ & $\mathrm{MW}=-1.13, \mathrm{p}=0.26$ \\
\hline Median width (mm, SD) & $18.0(3,4)$ & $18.6(3.7)$ & $\mathrm{MW}=-0.45, \mathrm{p}=0.65$ \\
\hline Median depth (mm, SD) & $4.1(1.7)$ & $4.3(1.6)$ & $\mathrm{MW}=-0.53, \mathrm{p}=0.60$ \\
\hline Distal width (mm, SD) & $15.0(2.4)$ & $15.6(3.1)$ & $\mathrm{MW}=-0.56, \mathrm{p}=0.56$ \\
\hline Distal depth (mm, SD) & $5.1(1.4)$ & $4,2(1.6)$ & $\mathrm{MW}=2,04, \mathrm{p}=0.04$ \\
\hline
\end{tabular}

Comparing surgical side to the contralateral one, we detected an increase of all measures in both groups (Table 5).

Table 5

Ultrasound measurements on operated tendon

\begin{tabular}{|llll|}
\hline & $\begin{array}{l}\text { Standard rehab. } \\
\mathbf{N}=18\end{array}$ & $\begin{array}{l}\text { Fast rehab. } \\
\mathbf{N}=15\end{array}$ & Average and P-value \\
\hline Proximal width (mm, SD) & $22.9(5.0)$ & $26.4(5.7)$ & $\mathrm{MW}=-0.90, \mathrm{p}=0.37$ \\
\hline Proximal depth (mm, SD) & $5.0(2.0)$ & $5.6(3.5)$ & $\mathrm{MW}=0.01, \mathrm{p}>0.95$ \\
\hline Median width (mm, SD) & $24.5(4.5)$ & $26.8(5.1)$ & $\mathrm{MW}=-1.32, \mathrm{p}=0.19$ \\
\hline Median depth (mm, SD) & $13.9(3.0)$ & $12.2(4.9)$ & $\mathrm{MW}=1.25, \mathrm{p}=0.21$ \\
\hline Distal width (mm, SD) & $18.0(2.2)$ & $20.4(2.9)$ & $\mathrm{MW}=-1.56, \mathrm{p}=0.12$ \\
\hline Distal depth (mm, SD) & $9.6(3.0)$ & $12.1(4.7)$ & $\mathrm{MW}=-1.48, \mathrm{p}=0.14$ \\
\hline
\end{tabular}

Using the ES, the percentage of stiffness on healthy side was increased at the proximal and medial third in patients treated with standard rehabilitation program, but on the distal third was major in patients underwent accelerated rehabilitation program. These differences were not statistically significant (Table 6).

Valuating the stiffness on the operated side, we observed that stiffness was more on the proximal third in patients treated with accelerate rehabilitation program but it was less on medial and distal third of AT (Table 7). All these differences were not statistically significant. 
Table 6

Elastosonography measurements on healthy tendon

\begin{tabular}{|llll|}
\hline & $\begin{array}{l}\text { Standard rehab. } \\
\mathbf{N}=\mathbf{1 8}\end{array}$ & $\begin{array}{l}\text { Fast rehab. } \\
\mathbf{N}=\mathbf{1 5}\end{array}$ & Average and P-value \\
\hline Proximal & $96.4 \%(12.1 \%)$ & $92.0 \%(16.1 \%)$ & $\mathrm{MW}=1.10, \mathrm{p}=0.27$ \\
\hline Median & $91.4 \%(11.6 \%)$ & $88.0 \%(11.3 \%)$ & $\mathrm{MW}=1.04, \mathrm{p}=0.30$ \\
\hline Distal & $82.8 \%(14.2 \%)$ & $86.3 \%(8.5 \%)$ & $\mathrm{MW}=-0.72, \mathrm{p}=0.47$ \\
\hline
\end{tabular}

Table 7

Elastosonography measurements on operated tendon

\begin{tabular}{|llll|}
\hline & $\begin{array}{l}\text { Standard rehab. } \\
\mathbf{N}=\mathbf{1 8}\end{array}$ & $\begin{array}{l}\text { Fast rehab. } \\
\mathbf{N}=1 \mathbf{1 5}\end{array}$ & Average and P-value \\
\hline Proximal & $90.0 \%(13.3 \%)$ & $91.3 \%(13.0 \%)$ & $\mathrm{MW}=-0.33, \mathrm{p}=0.74$ \\
\hline Median & $84.2 \%(10.0 \%)$ & $77.7 \%(16.5 \%)$ & $\mathrm{MW}=1.34, \mathrm{p}=0.18$ \\
\hline Distal & $78.6 \%(11.2 \%)$ & $74.3 \%(15.0 \%)$ & $\mathrm{MW}=0.61, \mathrm{p}=0.54$ \\
\hline
\end{tabular}

Then, we used the healthy side as a "basal" measure to compare the situation "before" and "after" surgery. We considered the healthy tendon measures comparable with those of the tendon operated before surgery.

We created a new variable comparing the US size of surgical and healthy side. If health value was $>0$ it meant that the size of tendon, detected with US, on the surgical side was major than the size on the healthy one, if it was $=0$ the size was the same on both sides and if it was $<0$ it meant that healthy side is bigger in size than the operated one. As expected, Table 8 show a general increase of tendon size on the surgical side compared to the "basal" one. The only statistically significant result was at distal third, where we detected an increase of depth of $7.9 \mathrm{~mm}$ with accelerate program compared with the $4.3 \mathrm{~mm}$ of the standard program $(p=0.01)$. 
Table 8

Variation in US measurements (average, $25^{\circ}$ and $75^{\circ}$ percentile)

\begin{tabular}{|llll|}
\hline Variation & $\begin{array}{l}\text { Standard rehab. } \\
\mathrm{N}=18\end{array}$ & $\begin{array}{l}\text { Fast rehab. } \\
\mathbf{N}=15\end{array}$ & Average and P-value \\
\hline Proximal width (mm, SD) & $2.8(-1.5 ; 6.0)$ & $2.8(1.8 ; 8.2)$ & $\mathrm{MW}=-0.20, \mathrm{p}=0.84$ \\
\hline Proximal depth (mm, SD) & $2.4(1.5 ; 3.7)$ & $2.2(0.0 ; 4.5)$ & $\mathrm{MW}=0.51, \mathrm{p}=0.61$ \\
\hline Median width (mm, SD) & $5.6(3.0 ; 10.7)$ & $8.3(5.8 ; 12.1)$ & $\mathrm{MW}=-1.25, \mathrm{p}=0.21$ \\
\hline Median depth (mm, SD) & $10.6(7.0 ; 12.1)$ & $7.6(4.0 ; 10.9)$ & $\mathrm{MW}=1.48, \mathrm{p}=0.14$ \\
\hline Distal width (mm, SD) & $4.3(0.5 ; 7.1)$ & $6.1(5.1 ; 10.0)$ & $\mathrm{MW}=-1.30, \mathrm{p}=0.19$ \\
\hline Distal depth (mm, SD) & $4.3(1.0 ; 7.2)$ & $7.9(5.0 ; 12.4)$ & $\mathrm{MW}=-2.46, \mathrm{p}=0.01$ \\
\hline
\end{tabular}

Also for the ES, we created a proxy of stiffness changes, using the differences between the surgical side and the healthy one (difference $>0$ meant that stiffness on surgical side was major than stiffness on the healthy side, difference $=0$ meant that stiffness was the same on both sides, difference $<0$ meant that there was less stiffness on the surgical side). After surgery we observed a general decrease in stiffness, but the same values grew up around the 12th month after surgery (Table 9). At the proximal side we observed a reduction of stiffness in patients treated with standard rehabilitation program (-5\%), while we didn't observe any differences in patients treated with accelerated rehabilitation program. At the medial third of the tendon we observed, in both groups of patients, a decreased stiffness (-10\%). At distal third of tendon we observed a greater reduction of stiffness in the accelerated group compared of the standard one.

Table 9

Variation in elastosonography measurements (average, $25^{\circ}$ and $75^{\circ}$ percentile)

\begin{tabular}{|llll|}
\hline Variation & $\begin{array}{l}\text { Standard rehab. } \\
\mathbf{N}=\mathbf{1 8}\end{array}$ & $\begin{array}{l}\text { Fast rehab. } \\
\mathbf{N}=\mathbf{1 5}\end{array}$ & Average and P-value \\
\hline Proximal & $-5.0 \%(-10.0 \% ; 0.0 \%)$ & $0.0 \%(-10.0 \% ; 0.0 \%)$ & $\mathrm{MW}=-0.79, \mathrm{p}=0.43$ \\
\hline Median & $-10.0 \%(-20.0 \% ; 0.0 \%)$ & $-10.0 \%(-30.0 \% ; 0.0 \%)$ & $\mathrm{MW}=0.48, \mathrm{p}=0.64$ \\
\hline Distal & $-5.0 \%(-15.0 \% ; 10.0 \%)$ & $-10.0 \%(-20.0 \% ; 0.0 \%)$ & $\mathrm{MW}=0.99, \mathrm{p}=0.32$ \\
\hline
\end{tabular}

Regarding the post-operative assessment scales of patients treated with the standard protocol compared to the accelerated one, the result is practically overlapping, as showed in Table 10. 
Table 10

Evaluation of post operative recovery with ATRS, VISA and AOFAS scales

\begin{tabular}{|llll|}
\hline & $\begin{array}{l}\text { Fast rehab. } \\
\mathbf{N}=\mathbf{1 5}\end{array}$ & $\begin{array}{l}\text { Standard } \\
\text { rehab. } \\
\mathbf{N}=18\end{array}$ & Average and P-value \\
\hline ATRS (average, SD) & $86.6(7.4)$ & $84.8(12.4)$ & $\mathrm{MW}=0.38, \mathrm{p}=0.70$ \\
\hline VISA(average, SD) & $86.2(15.7)$ & $85.2(14.7)$ & $\mathrm{MW}=0.33, \mathrm{p}=0.74$ \\
\hline AOFAS (average, SD) & $92.4(6.7)$ & $92.2(8.0)$ & $\mathrm{MW}=0.04, \mathrm{p}=0.97$ \\
\hline
\end{tabular}

\section{Discussion}

In previous literature [11], on average, the complete Achilles tendon injury is associated with a prolonged rehabilitation period (not less than 6 months) and a 10 to $30 \%$ sometimes permanent strength deficit.

Our early functional rehabilitation protocol is in line with the previous literature, including a variety of different exercise-based intervention. Concerning anearly recovery of full weight bearing, the clinical results are controversial [12]: in a systematic review, Mc Cormack et al. [13] concluded that early function rehabilitation, includes weight bearing initiated within first two weeks, is associated with higher patient satisfaction level, minimizing patients disability. Ankle range of motion was the most commonly included intervention [14] followed by progressive isometric strengthening of sural triceps. Many authors [14, 15] suggest that fast rehabilitation programs after open achilles tenorraphy allows to accelerate and facilitate the physiological repair process of the tendon suture, bringing a faster recovery of daily life activities and a return to pre-injury sports performance. Moreover, Maffulli et al. [16] reported that better satisfaction rate is due to shorter recovery time and less negative influence on daily life correlated with prolonged use of cast and immobilization.

In their study, Twaddle and Poon [17] suggest that the lower risk of recurrence and the apparently better outcome of surgical treatment compared to the conservative one can be attributed to the reduced immobilization that characterizes open surgery compared to the conservative method. They demonstrated that early mobilization affects both clinical outcome and relapse rates, as does surgery.

Kangas et al. [18] analysed fifty patients with acute Achilles tendon rupture which were randomized postoperatively to receive either early movement of the ankle between neutral and plantar flexion in a brace for 6 weeks of immobilization in tension using a below-knee cast with the ankle in a neutral position for 6 weeks. Tendon elongation occurred in both groups but was somewhat less in the early motion group (median $2 \mathrm{~mm}$ in the early motion group vs median $5 \mathrm{~mm}$ in the cast group a mean of 60 weeks postoperatively, $P=0.054)$. The elongation curves first rose and then slowly fell in both groups. The patients who had less elongation achieved a better clinical outcome $(P=0.042, P=0.017)$. The authors concluded that Achilles tendon elongation was somewhat less in the early motion group and 
correlated with the clinical outcome scores and recommend early functional postoperative treatment after Achilles rupture repair.

This generalized increased in volume, mostly on medial third of tendon, is due to surgical scar. Also we noticed that the proximal width, at the medial and distal regions, was increased in patients who underwent accelerated rehabilitation program compared to the ones treated with the standard one. The depth at the medial third, is the value who suffered more variation between the healthy tendon and the operated one, increase less with the accelerated rehabilitation program compared to the standard one.

Regarding the distal depth, in contrast to what observed on the healthy side, it increased in tendons treated with accelerated rehabilitation program but it is not statistically relevant.

The proximal, middle and distal third width in the case of patients treated with the accelerated protocol is higher than that of patients treated with the standard. Again, the fact that there is no statistically significant difference is due to the sample size. If the tendon is larger, it may mean that giving an accelerated mobilization stimulates more collagen deposition, this could be a protective factor against recurrence even if subjected to greater loads. The same reasoning can be performed on the proximal and distal depth values increased more with the accelerated method. On the other hand, the depth value in the middle third seems to contrast with the theory of greater collagen deposition as it appears to have increased less with the accelerated protocol compared to the standard one.

Although the results are not always uncontroversial between standard and accelerated protocols, in both cases the patients regain high levels of functional recovery. This evidence is due to the use of reliable surgical suture technique and development of suture material, combined with a rehabilitation program can be effective in reducing elongation of tendon and prevent re-rupture. At the time of data collect the patients had resumed work, the activities of daily life and completed the program for the return to sports. Regardless of the rehabilitation protocol it has been observed that the people who performed the postoperative physiotherapy more rigorously are also those who, as a rule, have the best outcome.

However accelerated protocol seems to provide positive aspects: first off all, according to Huang et al. [19] faster mobility of the ankle joint produced a better restoration of articular range of motion, with an improvement of local edema and to prevent stiffness and calf atrophy [20]. Moreover in previous literature [21] early weight bearing and faster recovery of ankle range of motion is linked to a reduction of some complications, as scarring adhesions, abnormal sensation and a fewer risk of deep vein thrombosis. The patient has to remove the brace several times during the day, so it is important recognize patient's compliance. The walker is easy to manage by the patient, the level of constriction of the brace can be regulated by straps and it makes him autonomous in removing wedges. The disadvantages of the accelerated protocol compared to the standard lie mainly in the fact that the patient has to bear the cost of purchasing the walker brace, as it is not provided by the NHS. Elective cases suitable for accelerated program rehabilitation are reliable patients who can join the rehabilitation program, especially to restore mobilization and muscles function. Young and sportive patient are theoretically ideal case, but it is important to underline that the compliance of the patient is mandatory to avoid suture dehiscence. 
Regarding clinical and functional outcomes, Ryu et al. [22] reported a case series of 112 patients treated with early rehabilitation for acute tear of Achilles tendons. All patients included in this study were fully satisfied, with an AOFAS score at 1 year follow up of 95.7. In a previous study, Kim [23] et al. compared functional results between a patients treated with an early rehabilitation protocol and patients treated with immobilization in cast. In this study the AOFAS score was slightly higher in the earlier group (93 compared to 89 of the cast group) with a statistically significant difference $(p<0.05)$. This result is comparable with the outcome reported in our series (92.2), although the difference is not statistically significant; this date is probably due to a low sample size of our study.

We agree with Porter and Shadbott [11] who demonstrated that an immediate mobilization after surgical Achilles tendon repair reduces tendon lengthening and time to return to sports, but it leads to similar Achilles tendon total rupture score relative to the standard rehabilitation. However, early functional rehabilitation has lacked a standardized definition, intervention and outcomes measures are highly variable [14].

After a surgical repair normally there is a lengthening of the tendon caused by separation of the tendon ends and by an elongation proximally and distally to the rupture site. The first matter can be reduced by a meticulous suture technique with appropriate suture wires; the second matter can be avoided, or reduced, by early mobilizations as evidenced by Kangas et al [15]. The authors showed in a randomized controlled trial that tendon elongation occurred both in early mobilization patients group both in immobilization patients group, but less in the early motion group and so this group of patient achieved a better clinical outcome. These evidences seem to favor adoption of this faster rehabilitation protocol especially in suitable and compliant patients. Benefits can be observed not only in terms of functional results but also in terms of tendon elasticity. Pathological processes at the level of the Achilles tendon, as for many other tissues, modify its physical characteristics of elasticity, viscoelasticity and mechanics. These modifications in turn influence the performance of the tendon causing the clinical symptoms. These alterations can be assessed through histological and biomechanical investigations which, however, as invasive, are not suitable for a clinical context such as post-surgery follow-up. So, ES has demonstrated to be a quick, relatively inexpensive, safe examination and therefore can be performed over time as many times as necessary without subjecting the patient to radiation. Furthermore, no special instruments are required but it is performed with a special ultrasound as it is equipped with additional software and hardware to determine the elasticity of the tissues, this factor brings both an economic and practical advantage for the radiologist who will not have to acquire additional skills. However, ultrasound is a method influenced by operator and it requires many hours of experience to be performed and interpreted in the most correct way.

The limits of the study are the limited sample size and the lack of randomization and double blindness. These last two points could have influenced the homogeneity of the patients assigned to the two clinicalrehabilitative treatments (standard protocol vs accelerated protocol) or have introduced potential prejudices in favor of one treatment rather than the other. 


\section{Conclusion}

Although no major statistically difference are reported in ES and US evaluation, clinical results and the previous literature evidence confirmed that a fast rehabilitation after Achilles tendons surgical repair may be a first choice of treat, especially in young and active patients. An early weight bearing and especially a quick recovery of ankle range of motion are associated with higher patient satisfaction level, minimizing patients' disability and local complications, as stiffness, edema or calf atrophy.

\section{Abbreviations}

AT

Achilles tendon

US

ultrasonography

ES

elastsonography

\section{Declarations}

Ethics approval, consent to participate: The institutional review board of the involved hospital decided that no ethical approval was necessary as it was thought that for this retrospective study the informed consent of the patient was sufficient.

Consent for publication: not applicable.

Availability of data and materials: The datasets used and analysed during the current study is available from the corresponding author on reasonable request.

Competing interests: The authors declare that they have no competing interests.

Authors' contribution: All the authors contributed in each part of this study.

Funding: The authors do not receive any found for this study.

Acknowledgements: not applicable.

\section{References}

1.

Lantto I, Heikkinen J, Flinkkila T, Ohtonen P, Leppilahti J. Epidemiology of Achilles tendon ruptures: increasing incidence over a 33-year period. Scand J Med Sci Sport. 2015 Feb;25(1):e133-8.

2. 
Longo UG, Rittweger J, Garau G, Radonic B, Gutwasser C, Gilliver SF. No influence of age, gender, weight, height and impact profile in Achilles tendinopathy in masters track and field athletes. Am J Sports Med. 2009;37:1400-5.

3.

Martorana,D.,Cannizzaro,E.,Tranchina,G.,Tranchina,E.,\&Martorana,V.Working tendinopatie: analysis of different surgical techniques and rehabilitation for rapid functional recovery in the subcutaneous injury of Achilles tendon.Acta Medica Mediterranea (2007),23(3),115-121.

4.

Bardas C, Benea H, Martin A, Tomoaia G. The traumatic rupture of the Achilles' tendon-an analysis of the modern methods of evaluation and treatment. Clujul Medical. 2015;86(2):128.

5 .

Gulati V, Jaggard M, Al-Nammari SS, Uzoigwe C, Gulati P, Ismail N, Gupte C. Management of achilles tendon injury: a current concepts systematic review. World journal of orthopedics. 2015;6(4):380.

6.

Kearney RS, McGuinness KR, Achten J, Costa ML. A systematic review of early rehabilitation methods following a rupture of the Achilles tendon. Physiotherapy. 2012;98(1):24-32.

7.

MaceraA,CarulliC,MatassiF,VenezianiC,InnocentiM.Traumatic bilateral Achilles tendon rupture in a young athlete treated with percutaneous tenorrhaphy.Joints (2015),3(04),218-220.

8.

SooHoo NF, Vyas R, Samini D. Responsiveness of the foot function index, AOFAS clinical rating systems, and SF-36 after foot and ankle surgery. Foot Ankle Int. 2006;27(11):930-4.

9 .

Iversen JV, Bartels EM, Langberg $\mathrm{H}$. The Victorian institute of sports assessment-Achilles questionnaire (VISA-A)-a reliable tool for measuring Achilles tendinopathy. International journal of sports physical therapy. 2012;7(1):76.

10.

Nilsson-Helander K, Thomeé R, Grävare-Silbernagel K, Thomeé P, Faxén E, Eriksson BI, Karlsson J. The Achilles tendon total rupture score (ATRS) development and validation. Am J Sports Med.

2007;35(3):421-6.

11.

Porter MD, Shadbolt B. Randomized controlled trial of accelerated rehabilitation versus standard protocol following surgical repair of ruptured Achilles tendon.ANZJ Surg (2015), 85373-377.

12.

Kearney RS. McGuinness KR, Achten J. Costa ML. A systematic review of early rehabilitation methods following a rupture of the Achilles tendon. Physiotherapy. 2012;98(1):24-32.

13.

McCormack R, Bovard J. Early functional rehabilitation or cast immobilization for the postoperative management of acute Achilles tendon rupture? A meta-analysis of randomized controlled trials. $\mathrm{Br} \mathrm{J}$ Sports Med. 2015;0:1-7. 
14.

Zellers JA, Christensen M, Kjær IL, Rathleff MS, Silbernagel KG. Defining Components of Early Functional Rehabilitation for Acute Achilles Tendon Rupture: A Systematic Review. Orthopaedic Journal of Sports Medicine 2019 Nov 25;7(11):2325967119884071.

15.

De la Fuente C, Peña y Lillo R, Carreño G, Marambio H. Prospective randomized clinical trial of aggressive rehabilitation after acute Achilles tendon ruptures repaired with Dresden technique. The Foot 2016 Mar;26:15-22.

16.

Maffulli N, Tallon C, Wong J, Lim KP, Bleakney R. Early weight-bearing and ankle mobilization after open repair of acute midsubstance tears of the Achilles tendon. Am J Sports Med. 2003;31:692-700.

17.

Twaddle BC, Poon P. Early motion for Achilles tendon ruptures: is surgery important? A randomized, prospective study. Am J Sports Med. 2007;35(12):2033-8.

18.

Kangas J, Pajala A, Ohtonen P, Leppilahti J. Achilles Tendon Elongation After Rupture Repair A Randomized Comparison of 2 Postoperative Regimens. The American Journal of Sports Medicine 2007, Vol. 35, No. 1.

19.

Huang J, Wang C, Ma X, Wang X, Zhang C, Chen L. Rehabilitation regimen after surgical treatment of acute Achilles tendon ruptures: a systematic review with meta-analysis. Am J Sports Med. 2015 Apr;43(4):1008-16.

20.

Khan RJ, Fick D, Keogh A, Crawford J, Brammar T, Parker M. Treatment of acute Achilles tendon ruptures: a meta-analysis of randomized, controlled trials. J Bone Joint Surg Am. 2005;87(10):2202-10. 21.

Costa ML, MacMillan K, Halliday D, et al. Randomised controlled trials of immediate weight-bearing mobilisation for rupture of the tendon Achillis. J Bone Joint Surg Am. 2006;88:69-77.

22.

Ryu CH, Lee HS, Seo SG, Kim HY. Results of tenorrhaphy with early rehabilitation for acute tear of Achilles tendon. Journal of Orthopaedic Surgery. 2018;26(3):1-7.

23.

Kim U, Choi YS, Jang GC, Choi YR. Early rehabilitation after open repair for patients with a rupture of the Achilles tendon. Injury. 2017;48(7):1710-3. 